\title{
Simultaneous Determination of 8 Compounds in Gancao-Ganjiang-Tang by HPLC-DAD and Analysis of the Relations between Compatibility, Dosage, and Contents of Medicines
}

\author{
Yanfang Yang, ${ }^{1}$ Guijun Zhang, ${ }^{1}$ Qiyu Sun, ${ }^{2}$ Liang Liu, ${ }^{2}$ Hui Peng, \\ Jingjuan Wang, ${ }^{1}$ and Li Xiang ${ }^{1}$ \\ ${ }^{1}$ Department of Pharmacognosy, Beijing University of Chinese Medicine, Beijing 100102, China \\ ${ }^{2}$ Zibo Wanjie Institute of Traditional Chinese Medicine, Shandong 255000, China \\ Correspondence should be addressed to Guijun Zhang; guijunzhang@163.com
}

Received 25 July 2016; Accepted 27 December 2016; Published 14 May 2017

Academic Editor: Pierre Champy

Copyright (C) 2017 Yanfang Yang et al. This is an open access article distributed under the Creative Commons Attribution License, which permits unrestricted use, distribution, and reproduction in any medium, provided the original work is properly cited.

\begin{abstract}
Gancao-Ganjiang-Tang (GGT) is a traditional Chinese medicine (TCM) prescription and is a representative prescription for recuperating depleted Yang in Treatise on Febrile Diseases. The TCM theory believes that the efficacy of medicinal herbs is decided by the multicompounds which consist of different kinds of chemical constituents with bioactivities, but not by a monomeric constituent. From ancient times until today, GGT have 5 different kinds of compatibilities that can be verified. In this study, a HPLC-DAD method was established for the simultaneous determination of 8 compounds including 6-gingerol, 8-gingerol, 6shogaol, liquiritin, liquiritigenin, isoliquiritin, isoliquiritigenin, and glycyrrhizic acid in the five GGT. The total contents of the 8 compounds in GGT varied from 555.56 to $956.33 \mu \mathrm{g} / \mathrm{mL}$. The effects showed that the dosage and compatibility of medicinal herbs have influenced the content of chemical compounds of TCM prescription while the content of chemical compounds has acted on clinical efficacy. Quality evaluation and active essence screening of TCM (including single herb and prescription) should be based on the TCM theory and clinical effectiveness. The method was proven to be suitable for quality control of GGT.
\end{abstract}

\section{Introduction}

Gancao-Ganjiang-Tang (GGT), a traditional Chinese medicine (TCM) prescription from the Treatise on Febrile Diseases, was a combination of the crude extracts from Glycyrrhizae Radix and Rhizoma Zingiberis and was used as a "warming" preparation for patients with chills, fatigue, dysphoric, and so forth caused by Yang deficiency [1]. GGT contains lots of chemical constituents and produces curative effects through synergic reaction. 6-Gingerol, 8-gingerol, 6-shogaol, liquiritin, liquiritigenin, isoliquiritin, isoliquiritigenin, and glycyrrhizic acid are the most important bioactive constituents reported at present. Glycyrrhizic acid can exert antiinflammation effect by suppressing cyclooxygenase-2 (COX2) and inducible nitric oxide synthase (iNOS) expression [2]; it also has other effects including immunomodulatory [3], antidiabetic effect [4], liver protection [5], inhibits cholestasis [6], and neuroprotection [7]. Liquiritigenin has the effects in reducing the expression of promatrix metalloproteinase2 [8] and inhibiting hypoxia-inducible factor $1 \alpha$ (HIF- $1 \alpha$ ) and vascular endothelial growth factor (VEGF) expression [9]. Isoliquiritigenin belongs to chalcone compound and has valuable biological activities such as anticancer, antioxidant, anti-inflammatory, and antiallergic activities. liquiritin and isoliquiritin have the pharmacological properties of antimicrobial [10], antioxidative [11], antitumor [12], and antiulcer activities [13] and others. Gingerols are a mixture and are the spicy ingredients in Rhizoma Zingiberis as well as the main active ingredients, among them are 6-gingerol, 8-gingerol, and 6-shogaol which are most outstanding. Gingerols can enhance power of cardiac muscle systole and have a protective effect on lipid peroxidation damage of nervous cell 
TABLE 1: Compatibility of medicines, dosage, and symptoms of GGT in different period.

\begin{tabular}{|c|c|c|c|c|}
\hline Reference & Period & Compatibility of TCM & Dosage & Symptoms \\
\hline $\begin{array}{l}\text { Shang-han-lun } \\
\text { (GGT-1) }\end{array}$ & Han dynasty & $\begin{array}{l}\text { Rhizoma Zingiberis (raw) and Radix } \\
\text { Glycyrrhizae (stir-baked with honey) }\end{array}$ & $12 \mathrm{~g}, 6 \mathrm{~g}$ & $\begin{array}{c}\text { Serious Yang } \\
\text { deficiency syndrome }\end{array}$ \\
\hline $\begin{array}{l}\text { Jin-kui-yao-lue } \\
\text { (GGT-2) }\end{array}$ & Han dynasty & $\begin{array}{l}\text { Rhizoma Zingiberis (stir-baked) and } \\
\text { Radix Glycyrrhizae (stir-baked with } \\
\text { honey) }\end{array}$ & $12 \mathrm{~g}, 6 \mathrm{~g}$ & $\begin{array}{l}\text { Relatively mild lung } \\
\text { deficiency }\end{array}$ \\
\hline $\begin{array}{l}\text { Ren-zhai-zhi-zhi- } \\
\text { fang-lun } \\
\text { (GGT-3) }\end{array}$ & Song dynasty & $\begin{array}{l}\text { Rhizoma Zingiberis (stir-baked) and } \\
\text { Radix Glycyrrhizae (stir-baked with } \\
\text { honey) }\end{array}$ & $9 \mathrm{~g}, 9 \mathrm{~g}$ & $\begin{array}{l}\text { Failure of spleen to } \\
\text { control circulating } \\
\text { blood caused by } \\
\text { spleen-Yang } \\
\text { deficiency }\end{array}$ \\
\hline $\begin{array}{l}\text { Xue-lun-zheng } \\
\text { (GGT-4) }\end{array}$ & Qing dynasty & $\begin{array}{l}\text { Rhizoma Zingiberis (stir-baked), Radix } \\
\text { Glycyrrhizae (stir-baked with honey), } \\
\text { and Schisandrae Chinensis Fructus (raw) }\end{array}$ & $15 \mathrm{~g}, 6 \mathrm{~g}, 3 \mathrm{~g}$ & $\begin{array}{l}\text { A variety of hernia } \\
\text { symptoms and } \\
\text { diarrhea }\end{array}$ \\
\hline $\begin{array}{l}\text { Shan-qi-zheng-zhi- } \\
\text { lun } \\
\text { (GGT-5) }\end{array}$ & Meiji restoration period (Japan) & $\begin{array}{l}\text { Rhizoma Zingiberis (raw), Radix } \\
\text { Glycyrrhizae (raw), Fructus Zanthoxyli } \\
\text { (raw), and Aconiti Lateralis Radix } \\
\text { Praeparata }\end{array}$ & $15 \mathrm{~g}, 15 \mathrm{~g}, 9 \mathrm{~g}, 9 \mathrm{~g}$ & Blood syndromes \\
\hline
\end{tabular}

membrane induced by radicals; gingerols can also effectively protect the activity of catalase in the ischemia reperfusion brain tissue of rats while simultaneously ameliorating the ischemia and anoxic state in tissue, and so forth [14]. All the compounds mixed together make the decoction have good efficacy in complex diseases.

Form ancient times, the combination of Rhizoma Zingiberis and Glycyrrhizae Radix has the remarkable curative effect to cold syndrome, such as epigastralgia, vomit acid saliva, diarrhea, back and chest pain, vertigo, and asthmatic cough, caused by Yang-qi deficiency and yin excessiveness [15]. The therapeutic rules for the Yang depletion are warming of the weakened organs and recuperating depleted Yang to save the body from collapsing [1]. The structure of Glycyrrhizae Radix and Rhizoma Zingiberis is a fundamental prescription for recuperating depleted Yang in TCM theories.

According to clinical symptoms, GGT have significant differences in compatibility and dosage (Table 1). For example, in Japan, GGT is composed of Glycyrrhizae Radix, Rhizoma Zingiberis, Fructus Zanthoxyli, and Aconiti Lateralis Radix Praeparata. It has been described in Shan-qi-zhengzhi-lun by Da-qiao-shang-yin in the Meiji Restoration, which is widely applied for the treatment of hernia symptoms and diarrhea. Similarly, GGT consists of Glycyrrhizae Radix, Rhizoma Zingiberis, and Schisandrae Chinensis Fructus in Xue-lun-zheng written by Rongchuan Tang and is aimed specifically at treating failure of spleen to control circulating blood. According to the theories of monarch drugs, minister drugs, assistant drugs, and guide drugs in TCM theories [16], the compatibility and dose of GGT have changed, but the Rhizoma Zingiberis and Glycyrrhizae Radix still dominate the curative powers.

TCM (including single herb and prescription) is a complex system, which contains many compounds. When single herb is used in combination, these compounds in each single herb will influence each other and then treat complex diseases together. Moreover, in TCM prescription, the change of dosage and compatibility of single herb may produce different symptoms. Now the quality control of TCM focuses on single herb or the monomeric compound, which cannot show the treatment effects accurately, cannot reflect the interactions between constituents, and cannot ensure the safety and efficacy of TCM. For example, both Chrysanthemi Flos and Lonicerae Japonicae Flos contain chlorogenic acid. Chlorogenic acid is regarded as marker for quality control of them in Chinese pharmacopoeia [17]. However, they are two different kinds of TCM with different clinical application.

Evaluating the quality of TCM should be based on TCM theory. The TCM theory believes the efficacy of TCM is decided by the multicompounds which consist of many different kinds of chemical constituents with bioactivities, but not by a monomeric constituent. In this study, a HPLCDAD method was developed to analyze multicompounds of GGT including 6-gingerol, 8-gingerol, 6-shogaol, liquiritin, liquiritigenin, isoliquiritin, isoliquiritigenin, and glycyrrhizic acid for exploring a comprehensive quality control criterion for TCM.

\section{Materials and Methods}

2.1. Chemicals and Reagents. The reference standards of 6-gingerol, 8-gingerol, 6-shogaol, liquiritin, liquiritigenin, isoliquiritin, isoliquiritigenin, and glycyrrhizic acid (purity $\geq 98 \%$ ) were purchased from Chengdu Pufeide Biotech Co., Ltd. (Chengdu, China). Methanol, acetonitrile (Fisher, USA), and phosphoric acid (Tianjin Chemical Regent Co., Ltd., Tianjin, China) were of HPLC grade. The distilled water was obtained from Wahaha Co., Ltd. (Hangzhou, China).

Medicinal materials were purchased from Hebei Anguo medicine market (Hebei, China) and authenticated by Professor Guijun Zhang (Beijing University of Chinese Medicine, Beijing, China).

2.2. Instrumentation and Separation Conditions. A thermo 3000 liquid chromatography system (Thermo, USA), equipped with a quaternary solvent delivery system, a DAD 
TABLE 2: The binary gradient elution system.

\begin{tabular}{|c|c|c|c|c|c|c|c|c|}
\hline Time (min) & $0 \sim 5$ & $5 \sim 10$ & $10 \sim 15$ & $15 \sim 30$ & $30 \sim 32$ & $32 \sim 35$ & $35 \sim 40$ & $40 \sim 45$ \\
\hline A: acetonitrile (\%) & $5 \sim 15$ & $15 \sim 22$ & $22 \sim 26$ & $26 \sim 28$ & $28 \sim 55$ & $55 \sim 60$ & $60 \sim 63$ & $63 \sim 65$ \\
\hline
\end{tabular}

TABLE 3: Detection wavelengths.

\begin{tabular}{|c|c|c|c|c|}
\hline Wavelengths (nm) & $235 \mathrm{~nm}$ & $254 \mathrm{~nm}$ & $280 \mathrm{~nm}$ & $370 \mathrm{~nm}$ \\
\hline Compounds & $\begin{array}{c}\text { Liquiritin } \\
\text { Liquiritigenin }\end{array}$ & Glycyrrhizic acid & $\begin{array}{l}\text { 6-Gingerol } \\
\text { 8-Gingerol } \\
\text { 6-Shogaol }\end{array}$ & $\begin{array}{c}\text { Isoliquiritin } \\
\text { Isoliquiritigenin }\end{array}$ \\
\hline
\end{tabular}

detector, an autosampler, a column heater, and a Hypersil Gold-C $\mathrm{C}_{18}(4.6 \mathrm{~mm} \times 250 \mathrm{~mm}, 5 \mu \mathrm{m})$ column, was used. The mobile phase consisted of (A) acetonitrile and (B) $0.1 \%$ $\mathrm{H}_{3} \mathrm{PO}_{4}$ aqueous (V/V). Optimum separation was obtained by using a gradient elution described in Table 2 . The flow rate was $0.6 \mathrm{~mL} \cdot \mathrm{min}^{-1}$ and injection volume was $20 \mu \mathrm{L}$. The column temperature was set at $30^{\circ} \mathrm{C}$ and the wavelengths were shown in Table 3.

2.3. Sample Preparation. TCM prescriptions (Table 1) were immersed with 10 -fold volume of water and boiled twice for $30 \mathrm{~min}$ at each time, followed by filtration. The filtrates from each prescription were merged and concentrated under reduced pressure to $1 / 2$ of its original volume. For HPLC analysis, $2 \mathrm{~mL}$ of filtrate was dissolved in methanol at a volume of $10 \mathrm{~mL}$ and then centrifuged with a rate of $100000 / \mathrm{min}$ for $10 \mathrm{~min}$. The supernatant was filtered through a filter $(0.22 \mu \mathrm{m}$ pore size) prior to injection. And the negative control groups were prepared in the same manner.

2.4. Preparation of Standard Solution. The appropriate amount of 6-gingerol, 8-gingerol, 6-shogaol, liquiritin, liquiritigenin, isoliquiritin, isoliquiritigenin, and glycyrrhizic acid was weighed and dissolved in methanol to achieve eight standard working solutions separately; the concentrations of the eight reference compounds were $1700 \mu \mathrm{g} / \mathrm{mL}, 870 \mu \mathrm{g} / \mathrm{mL}$, $960 \mu \mathrm{g} / \mathrm{mL}, 740 \mu \mathrm{g} / \mathrm{mL}, 810 \mu \mathrm{g} / \mathrm{mL}, 850 \mu \mathrm{g} / \mathrm{mL}, 770 \mu \mathrm{g} / \mathrm{mL}$, and $1110 \mu \mathrm{g} / \mathrm{mL}$. The calibration curves were constructed by analyzing the eight standard solutions and the series of working solutions within the ranges of $10.625 \sim 170 \mu \mathrm{g} / \mathrm{mL}$ for 6-gingerol, 5.4375 87 $\mu \mathrm{g} / \mathrm{mL}$ for 8-gingerol, $0.75 \sim 96 \mu \mathrm{g} / \mathrm{mL}$ for 6-shogaol, $18.5 \sim 296 \mu \mathrm{g} / \mathrm{mL}$ for liquiritin, $5.0625 \sim 81 \mu \mathrm{g}$ / $\mathrm{mL}$ for liquiritigenin, $7.9688 \sim 127.5 \mu \mathrm{g} / \mathrm{mL}$ for isoliquiritin, $0.9625 \sim 77 \mu \mathrm{g} / \mathrm{mL}$ for isoliquiritigenin, and $69.375 \sim 1110 \mu \mathrm{g} /$ $\mathrm{mL}$ for glycyrrhizic acid, respectively.

2.5. Method Validation. The HPLC method was employed and methodology was examined for linearity, recovery, precision, repeatability, and stability. The validation was implemented based upon the relative peak areas. Linear regression analysis was used to prepare calibration curves. And relative standard deviation (RSD) was used to evaluate precision, repeatability, stability, and recovery.

2.6. Statistical Analysis. Results were expressed as means \pm standard deviation (SD). Differences in mean values between groups were analyzed by a one-way analysis of variance (ANOVA). Statistical significance was considered at $P<0.05$.

\section{Results}

3.1. Chromatographic Conditions. Acetonitrile (A) and $0.1 \%$ $\mathrm{H}_{3} \mathrm{PO}_{4}$ aqueous (B) $(\mathrm{V} / \mathrm{V})$ were chosen as the composition of mobile phases for all the analyses. The chromatogram of mixed standard compounds, GGT sample, and negative control sample were shown in Figure 1. In Figure 1, the eight peaks marked with 1-8 are liquiritin, isoliquiritin, liquiritigenin, glycyrrhizic acid, isoliquiritigenin, 6-gingerol, 8-gingerol, and 6-shogaol. The retention time is $19.133 \mathrm{~min}, 26.300 \mathrm{~min}$, $30.767 \mathrm{~min}, 38.937 \mathrm{~min}, 39.487 \mathrm{~min}, 41.820 \mathrm{~min}, 46.797 \mathrm{~min}$, and $48.557 \mathrm{~min}$, respectively. The negative control sample had no peaks at the corresponding positions of GGT sample, which illustrated the other medicine herbs did not interfere the determination.

3.2. Calibration Curves. Using the above chromatographic conditions, the calibration curves of 8 compounds exhibited good linear regressions. The calibration curves were constructed by plotting the peak area and the corresponding concentration of the compounds in the freshly prepared plasma calibrators. Table 4 gives the linear regression equation, linear range, limit of quantitation (LOQs), and limit of detections (LODs) of eight standard substances.

3.3. Precision, Repeatability, Stability, and Recovery. The precision was obtained by six copies of determinations individually of the standard solution. The repeatability was performed by six-time determinations continuously of a sample. Stability was tested with GGT sample solution and standard solution that were stored at room temperature at several time points $(0,2,4,8,12$, and $24 \mathrm{~h}$ after preparation), and the 8 compounds were found to be rather stable within $24 \mathrm{~h}$ (RSD < $3 \% ; n=6)$. In the recovery test, samples were prepared at three concentration levels in triplicate by spiking known quantities of each of the 8 standards into the GGT sample and then extracted and analyzed according to the described procedures. All of these data are shown in Table 5.

3.4. Contents of Eight Compounds in GGT. The HPLC data illustrated that different compatibility of medicinal herbs has different contents. The material foundation of medicinal herbs is a combination of numerous active components 
TABLE 4: Linear regression equation, linear range, LOD, and LOQ for eight standard substances.

\begin{tabular}{|c|c|c|c|c|c|}
\hline Standard substance & Regression equation & Correlation coefficient $\left(r^{2}\right)$ & Linear range $(\mu \mathrm{g} / \mathrm{mL})$ & LOD $(\mu \mathrm{g} / \mathrm{mL})$ & LOQ $(\mu \mathrm{g} / \mathrm{mL})$ \\
\hline Liquiritin & $y=0.2557 x-0.5111$ & 0.9998 & $18.5 \sim 296$ & 1.52 & 4.56 \\
\hline Isoliquiritin & $y=1.6975 x-2.2464$ & 0.9999 & $7.9688 \sim 127.5$ & 0.512 & 1.536 \\
\hline Liquiritigenin & $y=1.3507 x-1.1905$ & 0.9997 & $5.0625 \sim 81$ & 0.477 & 1.431 \\
\hline Glycyrrhizic acid & $y=0.2929 x-3.2464$ & 0.9998 & $69.375 \sim 1110$ & 2.561 & 7.683 \\
\hline Isoliquiritigenin & $y=2.8843 x-0.2564$ & 0.9997 & $0.9625 \sim 77$ & 0.193 & 0.574 \\
\hline 6-gingerol & $y=0.2292 x-0.69$ & 0.9995 & $10.625 \sim 170$ & 1.847 & 5.541 \\
\hline 8-Gingerol & $y=0.2742 x-0.6137$ & 0.9997 & $5.4375 \sim 87$ & 1.013 & 3.039 \\
\hline 6-Shogaol & $y=2.4176 x-0.6731$ & 0.9996 & $0.75 \sim 96$ & 0.132 & 0.396 \\
\hline
\end{tabular}

TABle 5: Precision, repeatability, stability, and recovery of eight substances.

\begin{tabular}{|c|c|c|c|c|c|}
\hline \multirow[b]{2}{*}{ Compound } & \multirow{2}{*}{$\begin{array}{c}\text { Precision } \\
\text { RSD (\%) } \\
(n=6)\end{array}$} & \multirow{2}{*}{$\begin{array}{c}\text { Repeatability } \\
\text { RSD (\%) } \\
(n=6)\end{array}$} & \multirow{2}{*}{$\begin{array}{c}\text { Stability } \\
\text { RSD }(\%) \\
(n=6)\end{array}$} & \multicolumn{2}{|c|}{ Recoveries $(\%)^{*}$} \\
\hline & & & & $(n=9)$ & RSD (\%) \\
\hline Liquiritin & 0.88 & 1.36 & 0.51 & 99.89 & 1.58 \\
\hline Isoliquiritin & 1.02 & 1.19 & 0.79 & 101.67 & 1.28 \\
\hline Liquiritigenin & 1.73 & 0.97 & 1.32 & 98.93 & 1.33 \\
\hline Glycyrrhizic acid & 0.99 & 1.82 & 0.33 & 100.87 & 0.97 \\
\hline Isoliquiritigenin & 1.67 & 1.01 & 0.95 & 101.26 & 2.87 \\
\hline 6-Gingerol & 1.58 & 1.96 & 2.11 & 99.94 & 1.45 \\
\hline 8-Gingerol & 1.29 & 1.49 & 1.46 & 100.74 & 1.76 \\
\hline 6-Shogaol & 1.42 & 2.01 & 0.72 & 98.75 & 2.83 \\
\hline
\end{tabular}

${ }^{*}$ Recovery $(\%)=100 *($ amount found - original amount $) /$ amount spiked.

TABLE 6: The contents of eight compounds in GGT $(n=6)$.

\begin{tabular}{|c|c|c|c|c|c|}
\hline \multirow{2}{*}{ Compound } & \multicolumn{5}{|c|}{$C(\mu \mathrm{g} / \mathrm{mL})$} \\
\hline & GGT-1 & GGT-2 & GGT-3 & GGT-4 & GGT-5 \\
\hline Liquiritin & $175.68 \pm 0.932$ & $243.14 \pm 0.699$ & $151.42 \pm 0.865$ & $229.05 \pm 0.829$ & $144.81 \pm 0.687$ \\
\hline Isoliquiritin & $20.23 \pm 0.305$ & $29.87 \pm 0.108$ & $17.45 \pm 0.092$ & $28.06 \pm 0.42$ & $15.96 \pm 0.212$ \\
\hline Liquiritigenin & $10.87 \pm 0.068$ & $14.97 \pm 0.163$ & $8.92 \pm 0.053$ & $10.28 \pm 0.114$ & $11.23 \pm 0.086$ \\
\hline Glycyrrhizic acid & $367.79 \pm 0.987$ & $624.53 \pm 0.997$ & $344.03 \pm 1.212$ & $391.56 \pm 1.632$ & $366.93 \pm 1.230$ \\
\hline Isoliquiritigenin & $1.99 \pm 0.018$ & $3.29 \pm 0.012$ & $1.52 \pm 0.031$ & $2.23 \pm 0.011$ & $1.86 \pm 0.045$ \\
\hline 6-Gingerol & $29.48 \pm 0.114$ & $22.42 \pm 0.242$ & $24.21 \pm 0.346$ & $17.99 \pm 0.068$ & $40.89 \pm 0.920$ \\
\hline 8-Gingerol & $9.93 \pm 0.034$ & $14.97 \pm 0.162$ & $7.17 \pm 0.049$ & $10.89 \pm 0.052$ & $11.07 \pm 0.058$ \\
\hline 6-Shogaol & $4.88 \pm 0.029$ & $3.14 \pm 0.022$ & $0.84 \pm 0.015$ & $0.97 \pm 0.014$ & $1.29 \pm 0.011$ \\
\hline Total content of eight compounds & $620.85 \pm 1.42^{*}$ & $956.33 \pm 2.744$ & $555.56 \pm 1.23^{*}$ & $691.03 \pm 3.232$ & $594.04 \pm 3.41$ \\
\hline
\end{tabular}

${ }^{*}$ Compared with GGT-2, $P<0.05$.

following some complicated theorems (linear and nonlinear), rather than an element or a kind of element, or the simple sum of these elements. The content of eight compounds in GGT is shown in Table 6 and Figure 2.

\section{Discussion}

4.1. HPLC Conditions. Compared with acetonitrile and $0.05 \%$ phosphoric acid aqueous $(\mathrm{V} / \mathrm{V})$ and acetonitrile and $0.1 \%$ acetic acid aqueous (V/V), the separation ability of acetonitrile and $0.1 \%$ phosphoric acid aqueous (V/V) system is higher. The result showed that separation of peak 5 and peak 6 is not very good in $35^{\circ} \mathrm{C}$ and separation of peak 2 is not very good in $25^{\circ} \mathrm{C}$. By comparison, $30^{\circ} \mathrm{C}$ will be just right. The flow rate of $0.6 \mathrm{~mL} / \mathrm{min}$ was determined by testing different flow rates. When the flow rate is too high, such as $0.8 \mathrm{~mL} / \mathrm{min}$ and $1.0 \mathrm{~mL} / \mathrm{min}$, peaks cannot be separated from peaks. When the flow rate is too slow, the whole retention times were extended while the resolutions remained the same. The final gradient elution method was decided by testing various gradient elution methods.

4.2. Selections of Eight Active Compounds. The TCM theory believes that the efficacy of TCM is due to the multicompounds which consist of many different kinds of chemical constituents. Moreover, research and development of the multicompounds in TCM tend to be a direction of TCM modernization because of their therapeutic and 

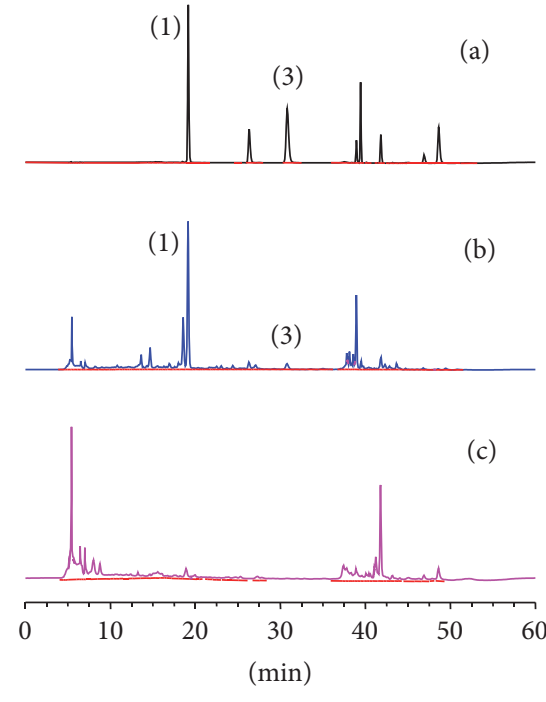

$\lambda=235 \mathrm{~nm}$
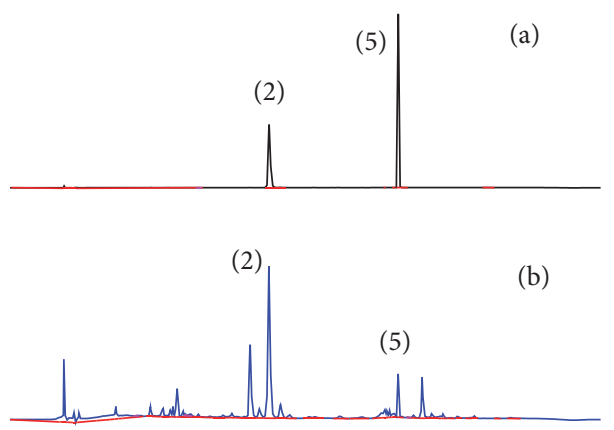

(c)

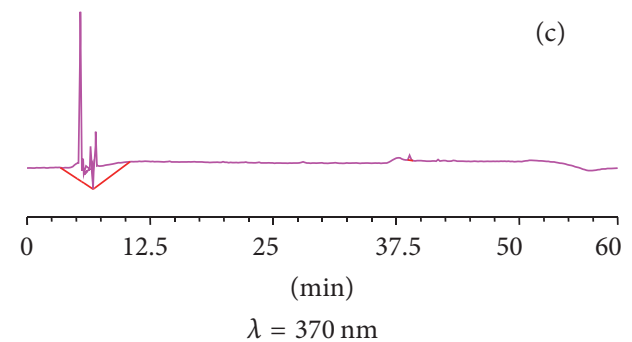

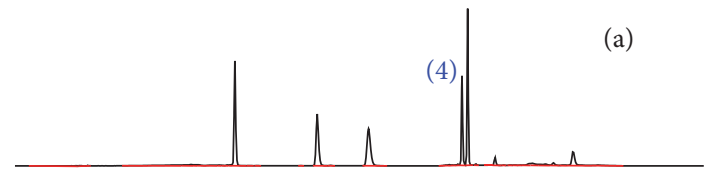

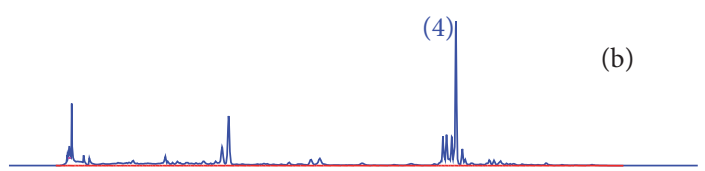

(c)

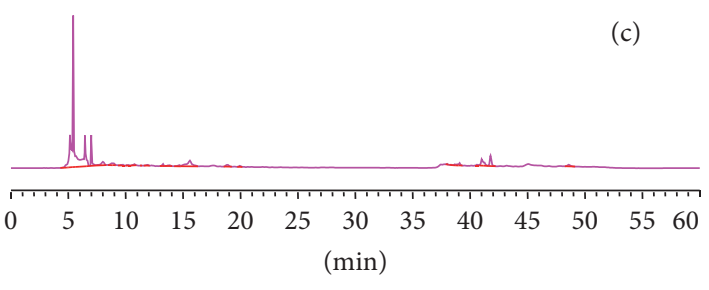

$\lambda=254 \mathrm{~nm}$

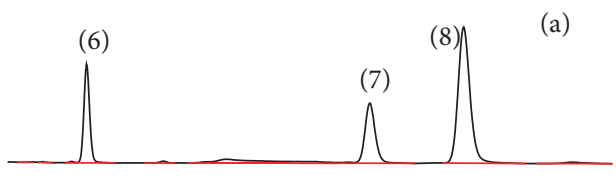

(b)

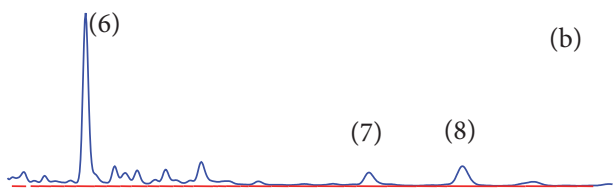

(d)

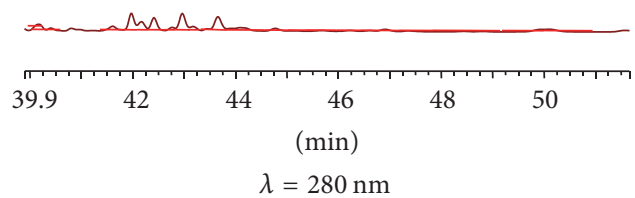

FIGURE 1: HPLC chromatograms of standard mixture (a), GGT test sample (b), test sample without Radix Glycyrrhizae (stir-baked with honey) (c), and test sample without Rhizoma Zingiberis (d). (1) Liquiritin, (2) isoliquiritin, (3) liquiritigenin, (4) glycyrrhizic acid, (5) isoliquiritigenin, (6) 6-gingerol, (7) 8-gingerol, and (8) 6-shogaol.

pharmaceutical advantages. 6-Gingerol, 8-gingerol, and 6shogaol are the major active components of Rhizoma Zingiberis [14]. Liquiritin, liquiritigenin, isoliquiritin, isoliquiritigenin, and glycyrrhizic acid are the major active components of Radix Glycyrrhizae [18]. The eight components of GGT have clear chemical structure, obvious pharmaceutical properties, and convenient detection, so they should be considered for study first.

4.3. Correlation between Compatibility, Dosage, and Content of Medicinal Herbs. According to the holistic concept and syndrome differentiation theories, it is a common phenomenon to change the TCM prescription (including compatibility and dosage) to accommodate symptoms. Meanwhile the compatibility and dosage of TCM prescription have an impact on its content of chemical compositions.

When the dosage of TCM prescription keeps constant and the compatibility of prescription changed, the content of components is changing with compatibility consequently. Take GGT-1 and GGT-2, for example: GGT-1 is composed of Rhizoma Zingiberis (6g, raw) and Radix Glycyrrhizae (12 g, stir-baked with honey), and GGT-2 is composed of Rhizoma Zingiberis (6 g, stir-baked) and Radix Glycyrrhizae (12 g, stir-baked with honey). The two prescriptions have same dosage but different medicinal herbs. We can see from Table 6 that the total content of eight compounds of GGT1 and GGT-2 had obvious difference and the difference was statistically significant $(P<0.05)$, which indicated that the 


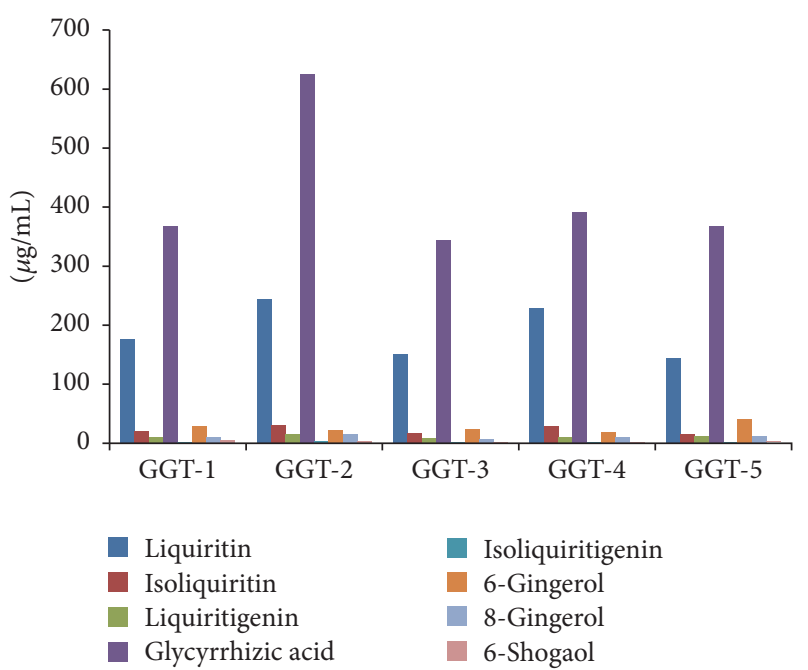

FIGURE 2: The contents of eight compounds in GGT $(\mu \mathrm{g} / \mathrm{mL})(n=$ 3).

compatibility has an impact on the content of medicinal herbs. Therefore GGT-1 and GGT-2 have different contents of chemical compounds. The contents are $(\mu \mathrm{g} / \mathrm{mL}) 175.68 \pm$ $0.932,20.23 \pm 0.305,10.87 \pm 0.068,367.79 \pm 0.987,1.99 \pm 0.018$, $29.48 \pm 0.114,9.93 \pm 0.034$ and $4.88 \pm 0.029$, and $243.14 \pm 0.699$, $29.87 \pm 0.108,14.97 \pm 0.163,624.53 \pm 0.997,3.29 \pm 0.012$, $22.42 \pm 0.242,14.97 \pm 0.162$, and $3.14 \pm 0.022$, respectively.

When the compatibility of TCM prescription keeps constant and the dosage of TCM prescription changed, the content of components is changing with dosage consequently. Take GGT-2 and GGT-3, for example: GGT-2 is composed of Rhizoma Zingiberis (6 g, stir-baked) and Radix Glycyrrhizae (12 g, stir-baked with honey), and GGT-3 is composed of Rhizoma Zingiberis ( $9 \mathrm{~g}$, stir-baked) and Radix Glycyrrhizae ( $9 \mathrm{~g}$, stir-baked with honey). The two prescriptions have the same compatibility but different dosage level. Similarly, the total content of eight compounds of GGT-2 and GGT-3 had obvious difference and the difference was statistically significant $(P<0.05)$, which indicated that the dosage has an impact on the content of medicinal herbs. Therefore GGT-2 and GGT-3 have the different contents of chemical compounds. The contents are $(\mu \mathrm{g} / \mathrm{mL}) 243.14 \pm 0.699,29.87 \pm$ $0.108,14.97 \pm 0.163,624.53 \pm 0.997,3.29 \pm 0.012,22.42 \pm 0.242$, $14.97 \pm 0.162$ and $3.14 \pm 0.022$, and $151.42 \pm 0.865,17.45 \pm 0.092$, $8.92 \pm 0.053,344.03 \pm 1.212,1.52 \pm 0.031,24.209 \pm 0.346$ $7.17 \pm 0.049$, and $0.84 \pm 0.015$, respectively.

From the above analysis, we can know when the compatibility and dosage of TCM prescription changed simultaneously and the content of components are changing consequently. Like with GGT-4 and GGT-5, GGT-4 is composed of Rhizoma Zingiberis (6 g, stir-baked), Radix Glycyrrhizae (15 g, stir-baked with honey), and Schisandrae Chinensis Fructus ( $3 \mathrm{~g}$, raw), and GGT-5 is composed of Rhizoma Zingiberis (15 g, raw), Radix Glycyrrhizae (15 g, raw), Fructus Zanthoxyli (9 g, raw), and Aconiti Lateralis Radix Praeparata $(9 \mathrm{~g})$. The two prescriptions have different compatibility and dosage level, and the effective substances of them are different: The contents of GGT- 4 are $(\mu \mathrm{g} / \mathrm{mL}) 229.05 \pm 0.829$,

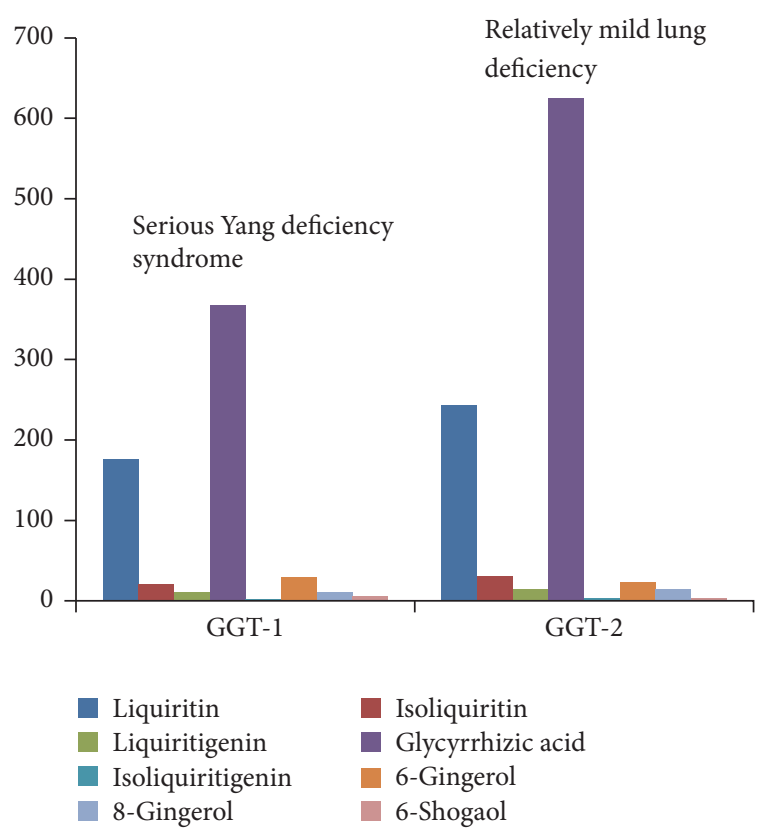

FIGURE 3: Correlation between symptoms and content of GGT-1 and GGT-2.

$28.06 \pm 0.42,10.28 \pm 0.114,391.56 \pm 1.632,2.23 \pm 0.011,17.99 \pm$ $0.068,10.89 \pm 0.052$ and $0.97 \pm 0.014$; GGT-5 are $(\mu \mathrm{g} / \mathrm{mL})$ $144.81 \pm 0.687,15.96 \pm 0.212,11.23 \pm 0.086,366.93 \pm 1.23$, $1.86 \pm 0.045,40.89 \pm 0.92,11.07 \pm 0.058$, and $1.29 \pm 0.011$.

4.4. Correlation between Compatibility, Dosage of Medicinal Herbs, and Symptoms. Traditional Chinese medicine decoction contains many compositions and various physicochemical actions coexist and interact. These compositions formed a relatively stable combination with a certain components and these components have a fixed ratio in content. This is why TCM prescription of different compatibility and dosage are being used to treat different diseases. The conclusion has been already proved and recorded in the medical classical documents written by medical experts at home and abroad in ancient times. GGT-1 is used to treat serious Yang deficiency syndrome, and symptoms include spontaneous perspiration, chills, dry throat, leg cramps, dizziness, vomit, and frequent urination, in Shang-han-lun by Zhongjing Zhang, while GGT-2 is applied to relatively mild lung deficiency, and symptoms include cough, dyspnea, and asthenia, in Jin-kui-yao-lue. And the composition ratios of them are $88: 10: 5: 184: 1: 15: 5: 2.5$ and $77: 9.5: 5: 199: 1: 7: 5: 1$, separately (Figure 3 ). GGT-4 is applied to failure of spleen to control circulating blood caused by spleen-Yang deficiency in Xue-lun-zheng by Rongchuan Tang; and GGT5 has specific effects and great power for a variety of hernia symptoms and diarrhea in Shan-qi-zheng-zhi-lun by Japanese scholar. And the composition ratios of them are $236: 29: 11: 404: 2: 19: 11: 1$ and $112: 12: 9: 284: 1: 32: 9: 1$, separately (Figure 4). GGT-3 is often used for blood syndromes, such as epistaxis, woman bleeding, and hematemesis in Ren-zhai-zhi-zhi-fang-lun by Shilei Yang. The composition ratio of GGT-3 is $180: 21: 11: 410: 2: 29: 9: 1$ (Figure 5). 


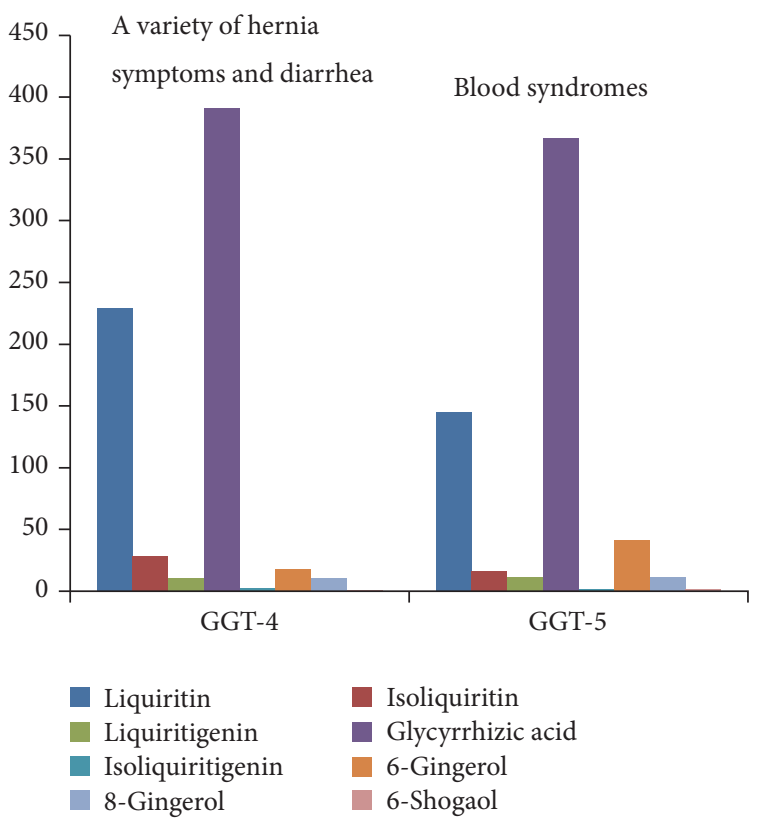

FIGURE 4: Correlation between symptoms and content of GGT-4 and GGT-5.

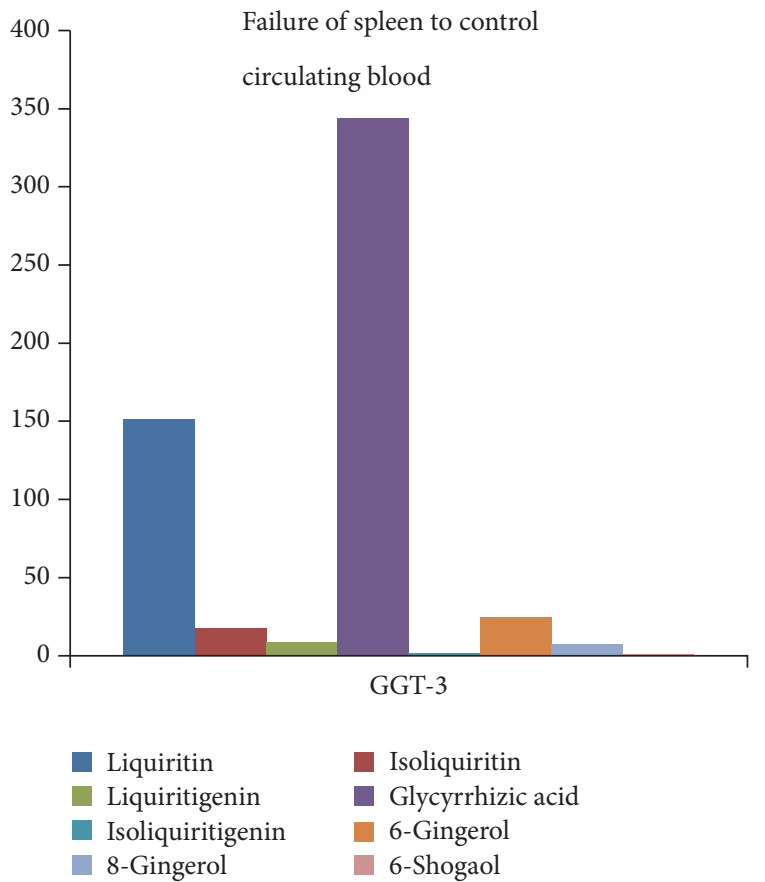

FIGURE 5: Correlation between symptoms and content of GGT-3.

\section{Conclusions}

TCM is the important part of Chinese culture, through thousands of years' development, having formed a selfcontained system and becoming the typical representative of eastern medicine. We should develop an effective and reasonable method to monitor the quality of TCM from a whole perspective. This study provides a new HPLC method for quality control of the GGT. The result showed that GGT mentioned above are different in compatibility and dosage, and the indications of them are different too. The method could be suitable for quality control of GGT with bioactive multicompounds.

\section{Competing Interests}

The authors declare no conflict of interests.

\section{Acknowledgments}

The authors acknowledge financial supports from the National Natural Science Foundation of China (no. 81303217).

\section{References}

[1] E. Zhang, Prescriptions of Traditional Chinese Medicine, Shanghai College of Traditional Chinese Medicine, Shanghai, China, 1990.

[2] Y.-F. Ni, J.-K. Kuai, Z.-F. Lu et al., "Glycyrrhizin treatment is associated with attenuation of lipopolysaccharide-induced acute lung injury by inhibiting cyclooxygenase- 2 and inducible nitric oxide synthase expression," Journal of Surgical Research, vol. 165, no. 1, pp. e29-e35, 2011.

[3] N. Bordbar, M. H. Karimi, and Z. Amirghofran, "The effect of glycyrrhizin on maturation and $\mathrm{T}$ cell stimulating activity of dendritic cells," Cellular Immunology, vol. 280, no. 1, pp. 44-49, 2012.

[4] S. Sen, M. Roy, and A. S. Chakraborti, "Ameliorative effects of glycyrrhizin on streptozotocin-induced diabetes in rats," Journal of Pharmacy and Pharmacology, vol. 63, no. 2, pp. 287296, 2011.

[5] C.-T. Tu, J. Li, F.-P. Wang, L. Li, J.-Y. Wang, and W. Jiang, "Glycyrrhizin regulates $\mathrm{CD} 4^{+} \mathrm{T}$ cell response during liver fibrogenesis via JNK, ERK and PI3K/AKT pathway," International Immunopharmacology, vol. 14, no. 4, pp. 410-421, 2012.

[6] D. Zhai, Y. Zhao, X. Chen et al., "Protective effect of glycyrrhizin, glycyrrhetic acid and matrine on acute cholestasis induced by $\alpha$-naphthyl isothiocyanate in rats," Planta Medica, vol. 73, no. 2, pp. 128-133, 2007.

[7] S.-W. Kim, Y. Jin, J.-H. Shin et al., "Glycyrrhizic acid affords robust neuroprotection in the postischemic brain via antiinflammatory effect by inhibiting HMGB1 phosphorylation and secretion," Neurobiology of Disease, vol. 46, no. 1, pp. 147-156, 2012.

[8] Y. Wang, S. Xie, C. Liu, Y. Wu, Y. Liu, and Y. Cai, "Inhibitory effect of liquiritigenin on migration via downregulation ProMMP-2 and PI3K/Akt signaling pathway in human lung adenocarcinoma A549 cells," Nutrition and Cancer, vol. 64, no. 4, pp. 627-634, 2012.

[9] S.-R. Xie, Y. Wang, C.-W. Liu, K. Luo, and Y.-Q. Cai, "Liquiritigenin inhibits serum-induced HIF- $1 \alpha$ and VEGF expression vi the AKT/mTOR-p70S6K signalling pathway in HeL cells," Phytotherapy Research, vol. 26, no. 8, pp. 1133-1141, 2012.

[10] A. Friis-Møller, M. Chen, K. Fuursted, S. B. Christensen, and A. Kharazmi, "In vitro antimycobacterial and antilegionella activity of licochalcone A from Chinese licorice roots," Planta Medica, vol. 68, no. 5, pp. 416-419, 2002. 
[11] H. Haraguchi, N. Yoshida, H. Ishikawa, Y. Tamura, K. Mizutani, and T. Kinoshita, "Protection of mitochondrial functions against oxidative stresses by isoflavans from Glycyrrhiza glabra," Journal of Pharmacy and Pharmacology, vol. 52, no. 2, pp. 219223, 2000.

[12] G. Yoon, B. Y. Kang, and H. C. Seung, "Topoisomerase I inhibition and cytotoxicity of licochalcones A and E from Glycyrrhiza inflata," Archives of Pharmacal Research, vol. 30, no. 3, pp. 313-316, 2007.

[13] B. Fuhrman, S. Buch, J. Vaya et al., "Licorice extract and its major polyphenol glabridin protect low-density lipoprotein against lipid peroxidation: in vitro and ex vivo studies in humans and in atherosclerotic apolipoprotein E-deficient mice," The American Journal of Clinical Nutrition, vol. 66, no. 2, pp. 267-275, 1997.

[14] S. Chrubasik, M. H. Pittler, and B. D. Roufogalis, "Zingiberis rhizoma: a comprehensive review on the ginger effect and efficacy profiles," Phytomedicine, vol. 12, no. 9, pp. 684-701, 2005.

[15] Y. Tang, "To explore therapeutic effect and clinical significance of Gancao-Ganjing," Journal of Practical Traditional Chinese Medicine, vol. 24, no. 3, pp. 190-191, 2008.

[16] F. Steven and C. Yue, Herbal Emissaries: Bringing Chinese Herbs to the West, Healing Arts Press, Rochester, Vt, USA, 1992.

[17] Chinese Pharmacopeia Commission, Pharmacopeia of People's Republic of China, vol. 1, China Medical Science and Technology Press, Beijing, China, 2015.

[18] P. P. Gan, X. Huang, M. Z. Zhong, M. Sun, F. Qin, and C. H. Zhang, "Simultaneous determination of eight major constituents in the traditional Chinese medicine ShaoyaoGancao-Tang by UPLC-PDA," Journal of Medicinal Plants Research, vol. 4, no. 24, pp. 2615-2621, 2010. 


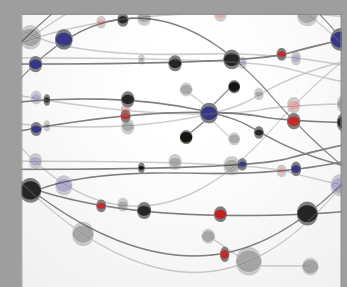

The Scientific World Journal
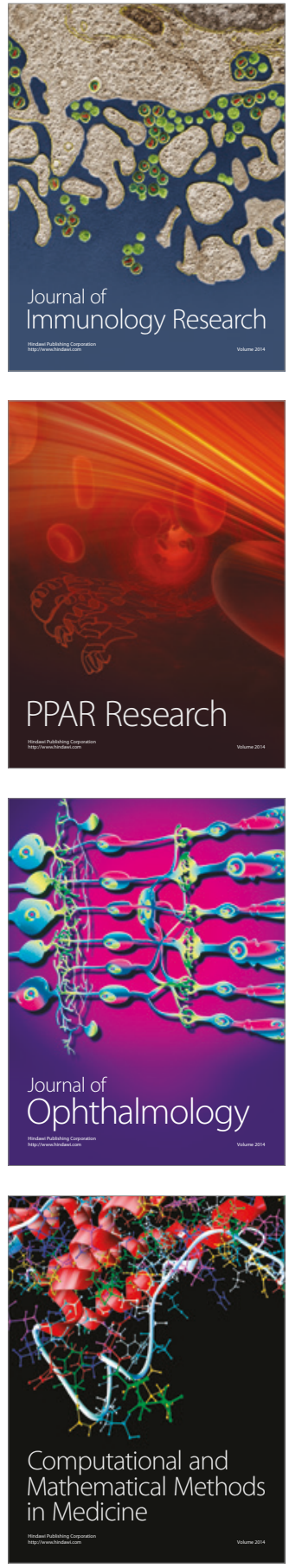

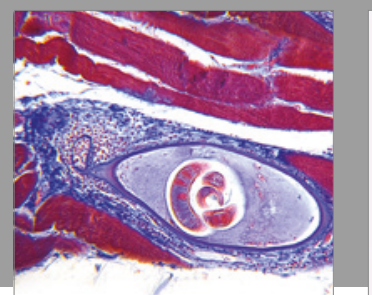

Gastroenterology Research and Practice
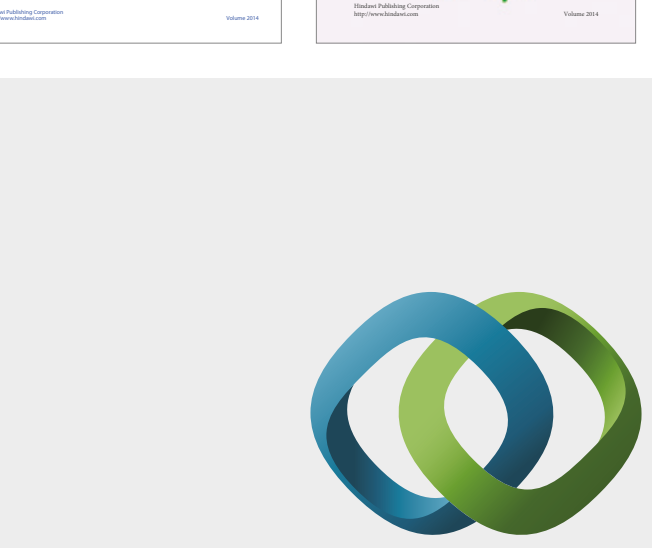

\section{Hindawi}

Submit your manuscripts at

https://www.hindawi.com
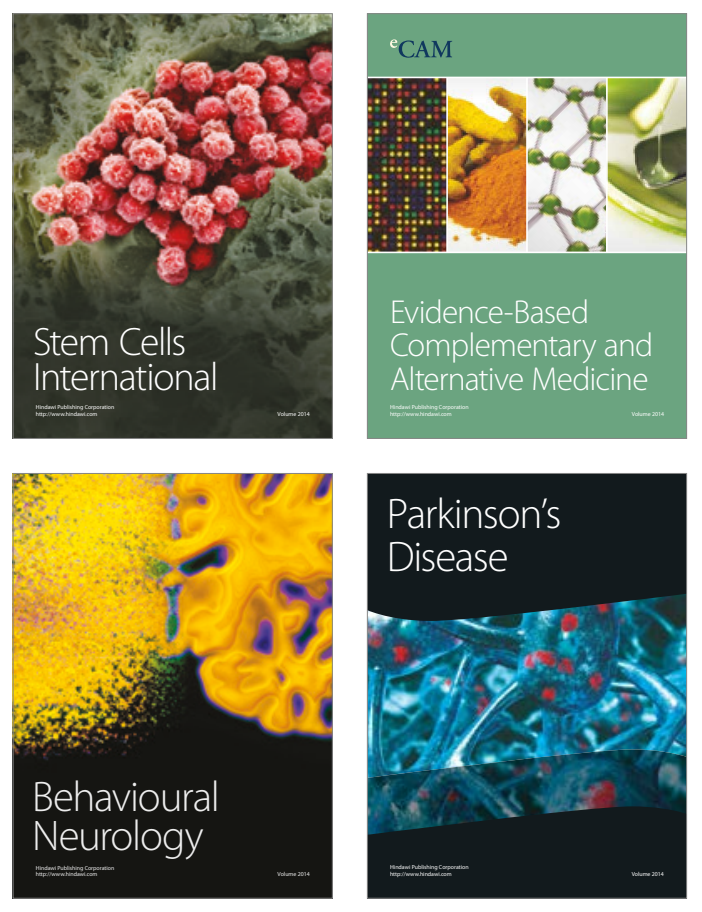
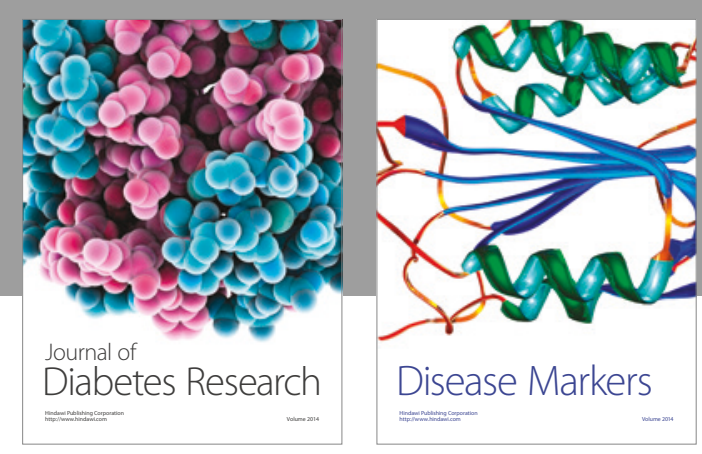

Disease Markers
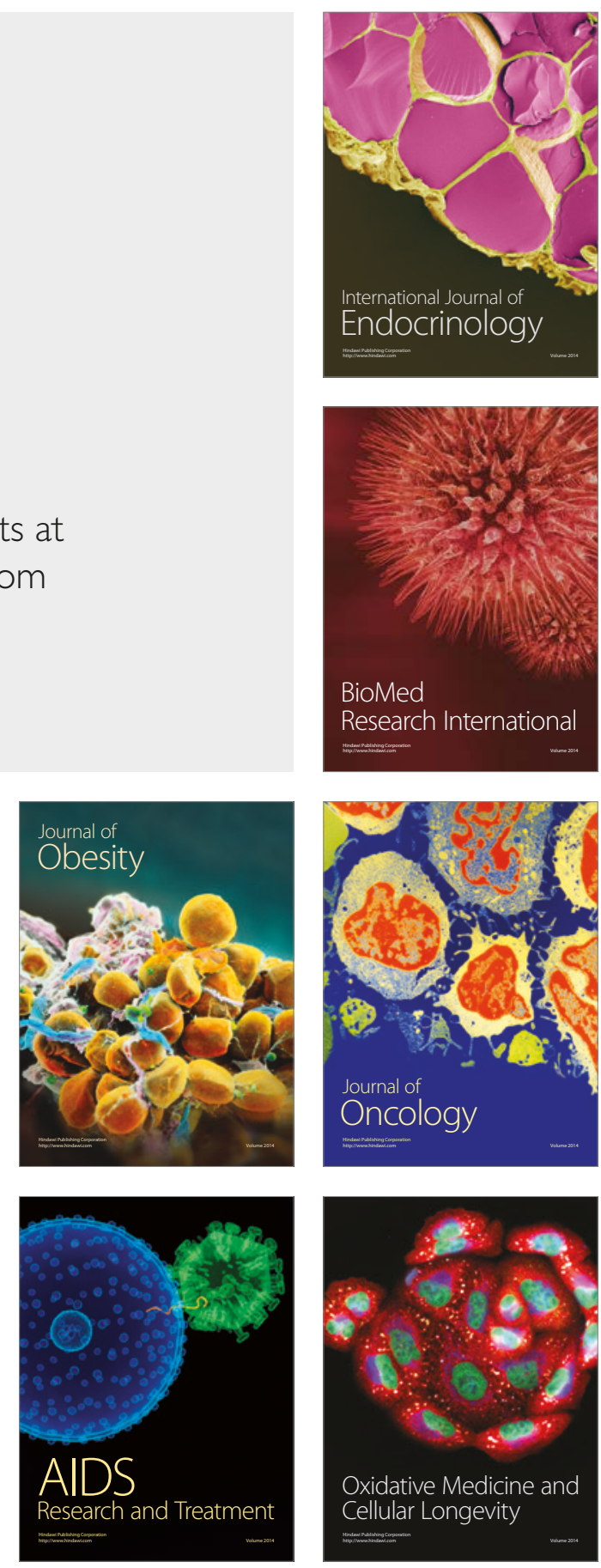